\title{
Evaluation of fatigue life of polycrystalline structural alloys
}

Ivan A. Volkov*, Leonid A. Igumnov, and Aleksandr A. Ipatov

Research Institute for Mechanics, National Research Lobachevsky State University of Niznhy Novgorod, Nizhny Novgorod, Russian Federation

\begin{abstract}
A mathematical model describing the processes of elastoplastic deformation and damage accumulation under low-cycle loading has been developed, based on the viewpoint of mechanics of damaged media (MDM). The MDM model consists of three interrelated parts: defining relations describing elastoplastic behavior of the materials, taking into account its dependence on the failure process; evolutionary equations describing the kinetics of damage accumulation; strength criteria of the damaged material. In order to assess the reliability and scope of applicability of the defining relations of mechanics of damaged media, the processes of plastic deformation and damage accumulation in variety of structural steels in low-cycle tests have been numerically analyzed, and numerical results obtained have been compared with the data of full-scale experiments. It is shown that the presented model of mechanics of damaged media adequately describes, both qualitatively and quantitatively, with accuracy, necessary for practical calculations, the main effects of the processes of plastic deformation and damage accumulation in structural alloys under block-type non-stationary non-symmetrical low-cycle loading.
\end{abstract}

\section{Introduction}

Cyclic properties of structural materials are of great importance for reliable evaluation of the strength and service life of elements and components of load-bearing structures under alternating combined thermo-mechanical effects. Calculation of the resource of structural elements based on the finite element analysis of inelastic deformations in hazardous zones of structural elements requires the formulation of the defining thermoplastic relations taking into account the real cyclic properties of materials [1].

Equations of state constructed on the basis of monotonous loading processes and not taking into account the peculiarities of cyclic deformation under proportional and disproportional loadings can lead to large errors in determining the basic parameters of the stress-strain state, which are then used to evaluate the resource characteristics of the material. The formulation of reliable defining thermoplastic relations for these processes requires, first of all, experimental studies of the effects of cyclic behavior of structural materials under proportional and disproportional loadings [2].

\footnotetext{
*Corresponding author: pmptmvgavt@yandex.ru
} 
The classical methods of predicting the fatigue life of materials using semi-empirical formulas (rules) based on a stabilized analysis of the deformation process and relating parameters of plastic hysteresis loops with the number of cycles prior to failure require a large amount of experimental deformation and are valid only for a narrow class of loading regimes within the available basic experimental data [3].

In recent years, to solve such problems, a new research area is being successfully developed - the mechanics of damaged medium (MDM) $[4,5]$.

\section{Defining relations of MDM}

The MDM model, developed in [4, 5], consists of three interrelated parts:

- relations defining elastoplastic behavior of materials, accounting for the effect of the failure process;

- evolutionary equations describing the kinetics of damage accumulation;

- strength criteria of the damaged material.

\subsection{Defining relations of plasticity}

Defining relations of plasticity [2] are based on the following main principals:

- components of stress tensors $e_{i j}$ and their velocities $\dot{e}_{i j}$ include elastic deformations $e_{i j}^{e}, \dot{e}_{i j}^{e}$ and plastic deformations $e_{i j}^{p}, \dot{e}_{i j}^{p}$;

- initial yeild surface for different temperatures is described using a Mises form of the yield surface. Evolution of change in yeild surface is described by the change in its radius $C_{p}$ and its centre $\rho_{i j}$;

- change in body volume is elastic;

- initially isotropic media are being considered. Only anisotropy due to plasticity processes is taken into account.

To describe the effects of monotonous and cyclic deformation, the yield surface is introduced in the form [2]:

$$
F_{s}=S_{i j} S_{i j}-C_{p}^{2}=0, S_{i j}=\sigma_{i j}^{\prime}-\rho_{i j} .
$$

To describe complex cyclic deformation modes in the stress space, a cyclic 'memory' surface is introduced:

$$
F_{\rho}=\rho_{i j} \rho_{i j}-\rho_{\max }^{2}=0,
$$

where $\rho_{\max }$ is maximum module in the loading history $\rho_{i j}$.

Evolutionary equation for the yield surface radius has the form [2]:

$$
\begin{gathered}
\dot{C}_{p}=\left[q_{\chi} H\left(F_{\rho}\right)+a\left(Q_{s}-C_{p}\right) \Gamma\left(F_{\rho}\right)\right] \dot{\chi}+q_{3} \dot{T} \\
C_{p}=C_{p}^{0}+\int_{0}^{t} \dot{C}_{p} d t, \dot{\chi}=\left(2 / 3 \dot{e}_{i j}^{p} \dot{e}_{i j}^{p}\right)^{1 / 2}, \chi_{m}=\int_{0}^{t} \dot{\chi} H\left(F_{\rho}\right) d t, \chi=\int_{0}^{t} \dot{\chi} d t . \\
q_{\chi}=\frac{q_{2} A \psi_{1}+(1-A) q_{1}}{A \psi_{1}+(1-A)}, Q_{s}=\frac{Q_{2} A \psi_{2}+(1-A) Q_{1}}{A \psi_{2}+(1-A)}, 0 \leq \psi_{i} \leq 1, i=1,2 . \\
A=1-\cos ^{2} \theta, \cos \theta=n_{i j}^{e} n_{i j}^{s}, n_{i j}^{e}=\frac{\dot{e}_{i j}^{\prime}}{\left(\dot{e}_{i j}^{\prime} \dot{e}_{i j}^{\prime}\right)^{1 / 2}}, n_{i j}^{s}=\frac{S_{i j}}{\left(S_{i j} S_{i j}\right)^{1 / 2}},
\end{gathered}
$$




$$
H\left(F_{\rho}\right)=\left\{\begin{array}{l}
1, F_{\rho}=0 \wedge \rho_{i j} \dot{\rho}_{i j}>0 \\
0, F_{\rho}<0 \vee \rho_{i j} \dot{\rho}_{i j} \leq 0
\end{array}\right\}, \Gamma\left(F_{\rho}\right)=1-H\left(F_{\rho}\right) .
$$

where $q_{1}, q_{2}, q_{3}$ are modules of isotropic hardening, $Q_{1}$ and $Q_{2}$ are modules of cyclic isotropic hardening, $a$ is constant, that determines the speed of the process of stabilization of the shape of the hysteresis loop of cyclic deformation of the material, $Q_{s}$ is stationary value of the yield surface radius for the given $\rho_{\max }$ and $T, C_{p}^{0}$ are initial values of the yield surface radius. We postulate that the evolution of an internal variable $\rho_{i j}$ is assumed in the form:

$$
\begin{gathered}
\dot{\rho}_{i j}=f\left(\chi_{m}\right)\left[g_{1} \dot{e}_{i j}^{p}-g_{2} \rho_{i j} \dot{\chi}\right]+g_{T} \rho_{i j}\langle\dot{T}\rangle+\dot{\rho}_{i j}^{*}, \rho_{i j}=\int_{0}^{t} \dot{\rho}_{i j} d t, f\left(\chi_{m}\right)=1+k_{1}\left(1-e^{-k_{2} \chi_{m}}\right), \\
\dot{\rho}_{i j}^{*}=g_{3} \dot{e}_{i j}^{p} H\left(F_{\rho}\right)-g_{4} \rho_{i j} \dot{\chi} \Gamma\left(F_{\rho}\right)\langle\cos \beta\rangle, \\
\langle\cos \beta\rangle=\frac{\dot{\rho}_{i j} \rho_{i j}}{\left(\dot{\rho}_{i j} \dot{\rho}_{i j}\right)^{1 / 2}\left(\rho_{i j} \rho_{i j}\right)^{1 / 2}},
\end{gathered}
$$

where $g_{1}, g_{2}, g_{3}, g_{4}, g_{T}, k_{1}$ and $k_{2}$ are experimentally determined material parameters. For asymmetric rigid and soft cyclic loading due to $\dot{\rho}_{i j}^{*}$ the equation (6) describes the processes of landing and "ratcheting" of the cyclic plastic hysteresis loop. At $g_{T}=g_{3}=g_{4}=k_{1}=0$ from (9) we obtain a special case of equation (6) - the Armstrong-Frederic-Kadashevich equation.

$$
\dot{\rho}_{i j}=g_{1} \dot{e}_{i j}^{p}-g_{2} \rho_{i j} \dot{\chi} .
$$

To characterize the behavior of the "memory" surface, it is necessary to formulate an evolution equation for $\rho_{\max }$ :

$$
\dot{\rho}_{\max }=\frac{\left(\rho_{i j} \dot{\rho}_{i j}\right) H\left(F_{\rho}\right)}{\left(\rho_{m n} \rho_{m n}\right)^{1 / 2}}-g_{2} \rho_{\max } \dot{\chi}-g_{T} \rho_{\max }\langle\dot{T}\rangle .
$$

At the stage of development of the scatted by volume damages, the effect of damage degree on the physico-mechanical properties of material is observed. This effect can be accounted by the introducing the effective stresses [4]:

$$
\begin{aligned}
& \tilde{\sigma}_{i j}^{\prime}=F_{1}(\omega) \sigma_{i j}^{\prime}=\frac{G}{\tilde{G}} \sigma_{i j}^{\prime}=\frac{\sigma_{i j}^{\prime}}{(1-\omega)\left[1-\frac{(6 K+12 G)}{(9 K+8 G)} \omega\right]}, \\
& \tilde{\sigma}=F_{2}(\omega) \sigma=\frac{K}{\tilde{K}} \sigma=\frac{\sigma}{4 G(1-\omega) /(4 G+3 K \omega)},
\end{aligned}
$$

where $\tilde{G}, \tilde{K}$ are effective elastic moduli determined by the McKenzie formulas [4].

The effective variable $\tilde{\rho}_{i j}$ is defined similarly:

$$
\tilde{\rho}_{i j}=F_{1}(\omega) \rho_{i j} .
$$




\subsection{Evolutionary equations of fatigue damage accumulation}

Let us postulate that the speed of damage accumulation under low cycle fatigue (LCF) is determined by an evolution equation of the form $[4,5]$ :

$$
\dot{\omega}=f_{1}(\beta) f_{2}(\omega) f_{3}(W) f_{4}(\dot{W}),
$$

where functions $f_{i}, i=1 \ldots 4$ account for volumetric character of the stress state $\left(f_{1}(\beta)\right)$, level of damage accumulation $\left(f_{2}(\omega)\right)$, accumulated relative energy of damage resulted in macrocrack formation $\left(f_{3}(W)\right)$ and rate of change in energy of damage $\left(f_{4}(\dot{W})\right)$.

In equation (14):

$$
\begin{gathered}
f_{1}(\beta)=\exp (\beta), f_{2}(\omega)=\left\{\begin{array}{l}
0, \quad W \leq W_{a} \\
\omega^{1 / 3}(1-\omega)^{2 / 3} \wedge W>W_{a} \wedge \omega \leq 1 / 3 \\
\frac{\sqrt[3]{16}}{9} \omega^{-1 / 3}(1-\omega)^{-2 / 3} \wedge W>W_{a} \wedge \omega>1 / 3
\end{array}\right. \\
f_{3}(W)=\frac{W-W_{a}}{W_{f}}, f_{4}(\dot{W})=\dot{W} / W_{f} .
\end{gathered}
$$

where $\beta$ is paremeter of the volumetric character of the stress state $\left(\beta=\sigma / \sigma_{u}\right), W_{a}$ is damage energy for the formation of scattered fatigue damage under low cycle fatigue (LCF); and $W_{f}$ is energy of microcrack formation.

\subsection{Criterion of strength of damaged material}

The condition when the damage degree $\omega$ reaches its critical value is taken as a criterion of the completion of the phase of development of scattered microcracks:

$$
\omega=\omega_{f} \leq 1 .
$$

\section{The investigation results}

Below are the results of experimental and theoretical studies of samples of 12X18H9 stainless steel under rigid non-stationary asymmetric cyclic loading consisting of two blocks:

- on the first block the sample is compressed until $e_{11}=0,01$ deformation, and then stretches to $e_{11}=0,05$ deformation;

- on the second block, asymmetric rigid cyclic loading is realized with a range of deformations $\Delta e_{11}=e_{11}^{(+)}-e_{11}^{(-)}=0,01$ up to $N_{f}=850$ destruction. Tables 1-3 presents the main physical and mechanical characteristics and material parameters of the MDM model used in the calculations for $12 \mathrm{X} 18 \mathrm{H} 9$ steel. 
Table 1. Physical-mechanical characteristics and parameters of MDM model of 12X18H9 stainless steel.

\begin{tabular}{|c|c|c|c|c|c|c|c|c|c|c|c|}
\hline$K$ & $G$ & $C_{p}^{o}, \mathrm{MPa}$ & $g_{1}, \mathrm{MPa}$ & $g_{2}$ & $g_{3}, \mathrm{MPa}$ & $g_{4}$ & $k_{1}, \mathrm{MPa}$ & $k_{2}$ & $a$ & $W_{a}$ & $W_{f}$ \\
\hline 165277 & 76282 & 190 & 24090 & 286 & 800 & 2 & 10000 & 0,2 & 5 & 0 & 800 \\
\hline
\end{tabular}

Table 2. Value of cyclic hardening modulus $Q_{S}\left(\rho_{\max }\right)(\mathrm{MPa}) 12 \mathrm{X} 18 \mathrm{H} 9$ stainless steel.

\begin{tabular}{|c|c|c|c|c|c|c|c|}
\hline$Q_{S}$, МПа & 190 & 205 & 210 & 215 & 220 & 225 & 225 \\
\hline$P_{\max }$, МПа & 0 & 20 & 40 & 60 & 80 & 100 & 120 \\
\hline
\end{tabular}

Table 3. Value of monotonous hardening modulus $q_{\chi}(\mathrm{MPa})$ of $12 \mathrm{X} 18 \mathrm{H} 9$ stainless steel.

\begin{tabular}{|c|c|c|c|c|c|c|c|c|c|c|c|c|}
\hline $\begin{array}{c}q_{\chi}, \\
\text { МП }\end{array}$ & -5000 & -4471 & -4188 & -3859 & -2460 & -182 & 888 & 1531 & 1274 & 913 & 913 & 913 \\
\hline$\chi$ & 0 & 0,002 & 0,004 & 0,006 & 0,008 & 0,01 & 0,015 & 0,02 & 0,03 & 0,04 & 0,05 & 0,06 \\
\hline
\end{tabular}

Fig. 1 depicts deformation process of $12 \mathrm{X} 18 \mathrm{H} 9$ stainless steel on the second loading block (the 500th cycle).

Fig. 2 shows the change in average cycle stress $\sigma_{11}^{(m)}$ under cyclic loading on the second block. It can be seen that a particular case of the thermoplastic model using defining relation for $\rho_{i j}$ in the form (9) implements the hysteresis loop immediately on the first loading cycle (dotted curve in Fig. 2), i.e. this variant of defining relations of plasticity can not describe the process of fitting of the cyclic hysteresis loop.

The model with the use of defining relation for $\rho_{i j}$ in the form (6) qualitatively and quantitatively describes the fitting process.

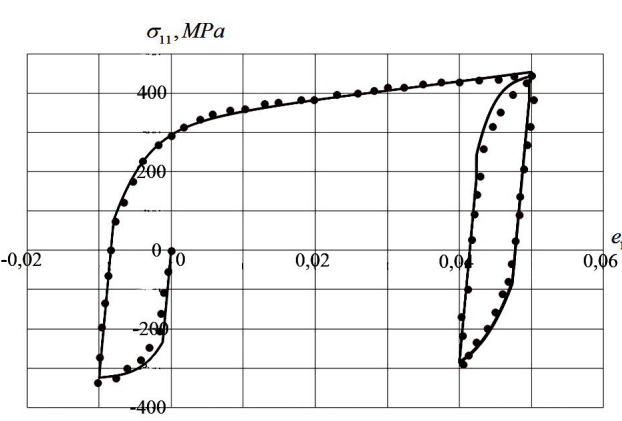

Fig. 1

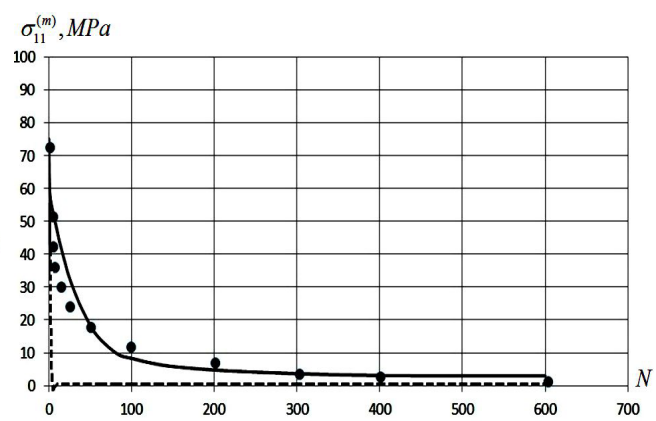

Fig. 2

Fig. 3 shows the fatigue curve of stainless steel $12 \mathrm{X} 18 \mathrm{H} 9$ with rigid symmetrical cyclic loading. Here the solid line shows the experimental curve, and the markers show the results of calculations using defining relations of the MDM. In Fig. 3 round marks show the calculated data of thermoplasticity model using the defining relations for $\rho_{i j}$ in the form (6), and square markers show the data using the ratio for $\rho_{i j}$ in the form (9). It can be seen that the calculations of fatigue life on both models almost coincide. 


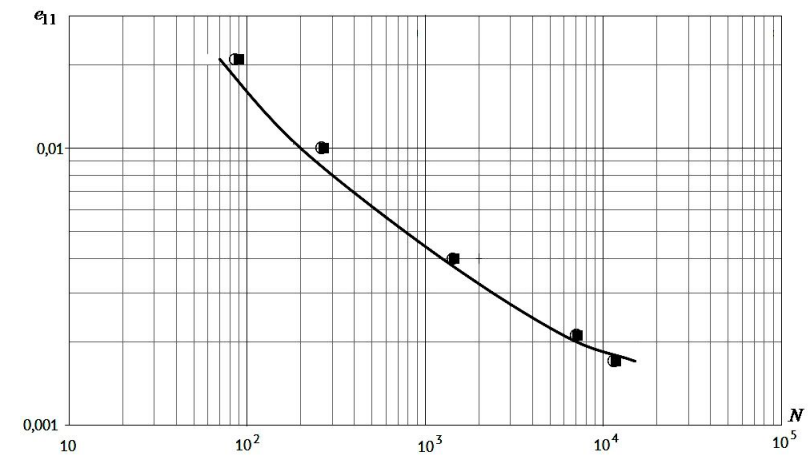

Fig. 3. the fatigue curve of stainless steel $12 \mathrm{X} 18 \mathrm{H} 9$ with rigid symmetrical cyclic loading.

\section{Conclusion}

To describe the fatigue life of polycrystalline structural alloys under block asymmetric lowcycle loading, the reliability of defining relations of the MDM has been assessed by comparing the results of numerical experiments with experimental data on plastic deformation and damage accumulation in 12X18H9 steel under block unsteady asymmetric low-cycle loading, which corroborated the adequacy of the modeling and determining material parameters .

The work is financially supported by the Federal Targeted Program for Research and Development in Priority Areas of Development of the Russian Scientific and Technological Complex for 2014-2020 under the contract No. 14.578.21.0246 (unique identifier RFMEFI57817X0246).

\section{References}

1. F. M. Mitenkov, V. B. Kaidalov, Yu. G. Korotkhih etc. Methods of justification of resource NPIs. (Machine Building, Moscow, 2007)

2. F. M. Mitenkov, I. A. Volkov, L. A. Igumnov, Yu. G. Korotkhih etc. Applied theory of plasticity (Fizmatlit, Moscow, 2015)

3. J. Collins Damage to materials in structures. Analysis. Prediction. Prevention. (World, Moscow, 1984)

4. I. A. Volkov, L. A. Igumnov Introduction to the continuum mechanics of the damaged medium (Fizmatlit, Moscow, 2017)

5. I. A. Volkov, Yu. G. Korotkhih The Equation of state viscoplasticity media damage (Fizmatlit, Moscow, 2008) 